\title{
UCRL-CONF-216863
}

LAW RENCE LIVERMORE N A T IO N A L LABORATORY

\section{A multi-dimensional investigation of laser conditioning in KDP and DKDP crystals}

P. DeMange, R. A. Negres, C. W. Carr, H. B. Radousky, S. G. Demos

November 4, 2005

Boulder Damage Symposium

Boulder, CO, United States

September 19, 2005 through September 21, 2005 
This document was prepared as an account of work sponsored by an agency of the United States Government. Neither the United States Government nor the University of California nor any of their employees, makes any warranty, express or implied, or assumes any legal liability or responsibility for the accuracy, completeness, or usefulness of any information, apparatus, product, or process disclosed, or represents that its use would not infringe privately owned rights. Reference herein to any specific commercial product, process, or service by trade name, trademark, manufacturer, or otherwise, does not necessarily constitute or imply its endorsement, recommendation, or favoring by the United States Government or the University of California. The views and opinions of authors expressed herein do not necessarily state or reflect those of the United States Government or the University of California, and shall not be used for advertising or product endorsement purposes. 


\title{
A multi-dimensional investigation of laser conditioning in KDP and DKDP crystals
}

\author{
P. DeMange, R. A. Negres, C. W. Carr, H. B. Radousky, and S. G. Demos \\ Lawrence Livermore National Laboratory, 7000 East Avenue, Livermore, California 94551
}

\begin{abstract}
We present a multi-parametric experimental investigation of laser conditioning efficiency and behavior in KDP and DKDP crystals as a function of laser wavelength, fluence, number of pulses, and conditioning protocol. Our results expose complex behaviors associated with damage initiation and conditioning at different wavelengths that provide a major step towards revealing the underlying physics. In addition, we reveal the key parameters for optimal improvement to the damage performance from laser conditioning.
\end{abstract}

\section{INTRODUCTION}

Laser-induced bulk damage sites arising in KDP and DKDP optics used in large-aperture laser systems is a significant deterrent to performance. The damage precursors are believed to be clusters of intrinsic defects and/or nano-particle impurities, however, identifying them has been difficult due to their size and sparse distribution. Laser conditioning is believed to be the result of the interaction of DKDP/DKDP defects (damage initiators) with sub-damage laser intensities in which the defects are annealed or modified thus providing an increase to the material damage threshold. The mechanisms leading to conditioning of these defects and the operational parameters that can optimize the effect for application on large-aperture laser systems are largely unknown. In this work, we measure the damage behaviors in preexposed DKDP/DKDP applying several protocols and variable excitation conditions to investigate the fundamental mechanisms of laser conditioning. The results provide important results regarding the nature of the defects responsible for damage initiation and reveal two distinct pathways leading to conditioning that depend on the pre-exposure fluence and wavelength. Moreover, these results reveal the laser parameters and protocols necessary for optimizing conditioning.

\section{EXPERIMENTAL SETUP}

The experimental arrangement has been described in detail elsewhere ${ }^{1}$ but the basic setup and approach is described here briefly. The conditioning experiments are performed using a pulsed-Nd:YAG laser. The fundamental (at $1064 \mathrm{~nm}$ ), second, and third harmonics of the output are separated using high reflectivity mirrors selective to each wavelength. The average fluence of each beam is adjusted using a waveplate and a polarizer. The three beams are then aligned to copropagate and focused by a $200-\mathrm{mm}$ focal length cylindrical lens to the bulk of the sample. The beams focus to a $1 / \mathrm{e}^{2}$ height of $3 \mathrm{~mm}$ and widths of $90 \mu \mathrm{m}, 60 \mu \mathrm{m}$, and $40 \mu \mathrm{m}$ for 1064, 532 and $355 \mathrm{~nm}$, respectively. In order to focus each wavelength to the same location in the bulk of the crystal, we have positioned galilean telescopes in each beam line to adjust their divergence before recombination. The beam spatial profiles are measured using a 0.25 by $0.25 \mathrm{inch}^{2} \mathrm{CCD}$ camera with a pixel resolution of $\sim 5 \mu \mathrm{m}$.

The samples were cut to $1 \times 5 \times 5 \mathrm{~cm}^{3}$ size plates and polished on all sides. Bulk damage is produced through the focal range of the cylindrical lens, $\sim 8 \mathrm{~mm}$. A counter-propagating $632.8-\mathrm{nm}$ beam from a HeNe laser is focused by a 250-mm focal length cylindrical lens through the back of the sample to illuminate any resulting damage pinpoints in the tested volume. Images of the damaged regions are captured orthogonally to the direction of propagation of the lasers, through the side of the sample. A \% 2 magnification microscope objective followed by $\% 5$ magnification lens is used that provides a $4.7 \mu \mathrm{m}$ by $4.7 \mu \mathrm{m}$ per pixel image resolution. Figure 1 shows captured images of scatter from pinpoint damage sites in bulk DKDP that demonstrate both a typical damage measurement and the manifestation of the 
conditioning effect using this approach. The images capture $5.9 \mathrm{~mm}$ in length of the damaged volume along the direction of propagation of the beam. Figure 1a shows damage resulting from a single pulse at $15 \mathrm{~J} / \mathrm{cm}^{2}$ in pristine material and Fig. $1 \mathrm{~b}$ shows damage resulting from a single pulse at $15 \mathrm{~J} / \mathrm{cm}^{2}$ following pre-exposure to ten pulses at $10 \mathrm{~J} / \mathrm{cm}^{2}$ for conditioning. The damage pinpoint density, or PPD, is measured over the volume exposed to peak laser fluence $( \pm 5 \%)$ which is the region enclosed by the white lines shown in each image. Comparison of the two images demonstrates that there is significantly less damage in Fig. $1 \mathrm{~b}\left(\sim 150 \mathrm{pp} / \mathrm{mm}^{3}\right)$ than in Fig. 1a $\left(\sim 1180 \mathrm{pp} / \mathrm{mm}^{3}\right)$ as a result of having preexposed the material to the ten pulses.

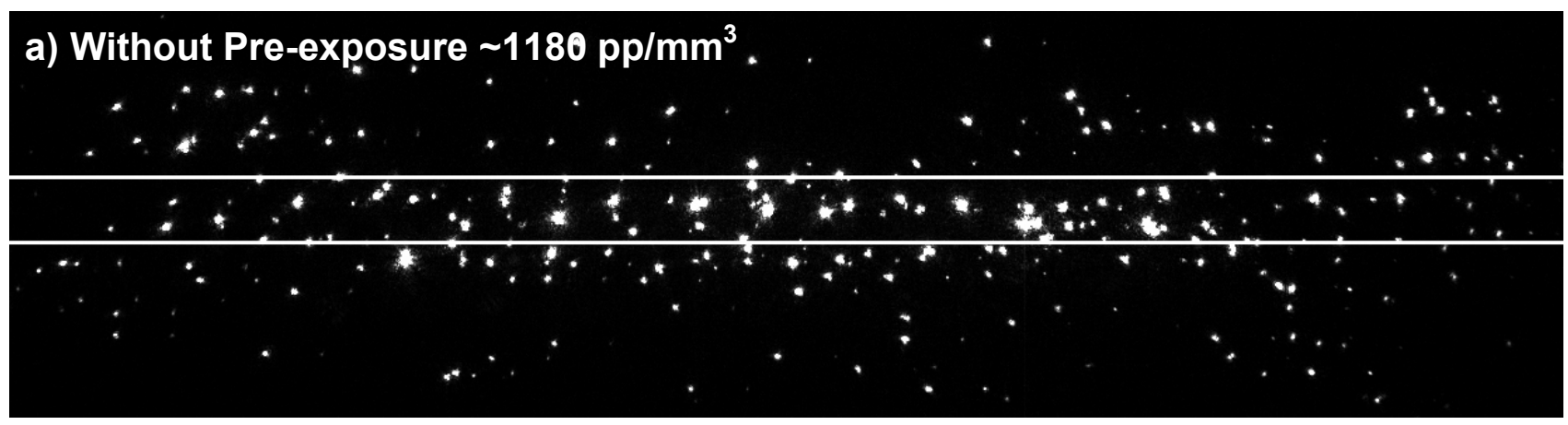

\section{b) With Pre-exposure $\sim 150 \mathrm{pp} / \mathrm{mm}^{3}$}

Figure 1. Scatter images of pinpoint damage sites resulting from exposure to a) one pulse at $15 \mathrm{~J} / \mathrm{cm}^{2}$ in pristine material $(\sim 1180$ $\left.\mathrm{pp} / \mathrm{mm}^{3}\right)$ and $\mathrm{b}$ ) one pulse at $15 \mathrm{~J} / \mathrm{cm}^{2}$ following pre-exposure to ten pulses at $10 \mathrm{~J} / \mathrm{cm}^{2}\left(\sim 150 \mathrm{pp} / \mathrm{mm}^{3}\right)$. The PPD is measured over the volume exposed to peak laser fluence (region enclosed by the white lines).

\section{EXPERIMENTS AND RESULTS}

The damage performance of DKDP is investigated following pre-exposure of the material to laser pulses under variable excitation conditions including wavelength, fluence, and number of damage testing and pre-exposure pulses. We explore several conditioning protocols in order to reveal fundamental behaviors associated with the conditioning and damage initiation of the defect damage precursors as well as learn how to optimally condition the material.

\subsection{Conditioning vs. Pre-exposure Fluence and Damage Testing and Pre-exposure Wavelength}

The dependence of conditioning on pre-exposure fluence was measured at combinations of damage testing and preexposure wavelengths at 1064, 532, and $355 \mathrm{~nm}$. Pristine bulk sites in conventional-growth DKDP were exposed first to ten pulses of the same fluence and then to a single higher fluence damage testing pulse. The fluence of the damage testing pulse remained the same for all sites $\left(46 \mathrm{~J} / \mathrm{cm}^{2}\right.$ for $1064 \mathrm{~nm}, 31 \mathrm{~J} / \mathrm{cm}^{2}$ for $532 \mathrm{~nm}$, and $23 \mathrm{~J} / \mathrm{cm}^{2}$ for $355 \mathrm{~nm}$ ) while the fluence of the ten pre-exposure pulses was varied. Images were recorded prior to and after irradiation at a high fluence and the PPD at peak fluence resulting from the damage testing pulse only was plotted versus the peak preexposure fluence. 
Figures $2 \mathrm{a}-2 \mathrm{c}$ show the density of new damage pinpoints resulting from single-pulse damage testing at $46 \mathrm{~J} / \mathrm{cm}^{2}$ at $1064 \mathrm{~nm}, 31 \mathrm{~J} / \mathrm{cm}^{2}$ at $532 \mathrm{~nm}$, and $23 \mathrm{~J} / \mathrm{cm}^{2}$ at $355 \mathrm{~nm}$, respectively, as a function of the fluence of the ten pre-exposure pulses. There are three sets of data in each figure corresponding to pre-exposure at each of the three harmonics. It must be noted that these figures do not include the PPD resulting from pre-exposure.
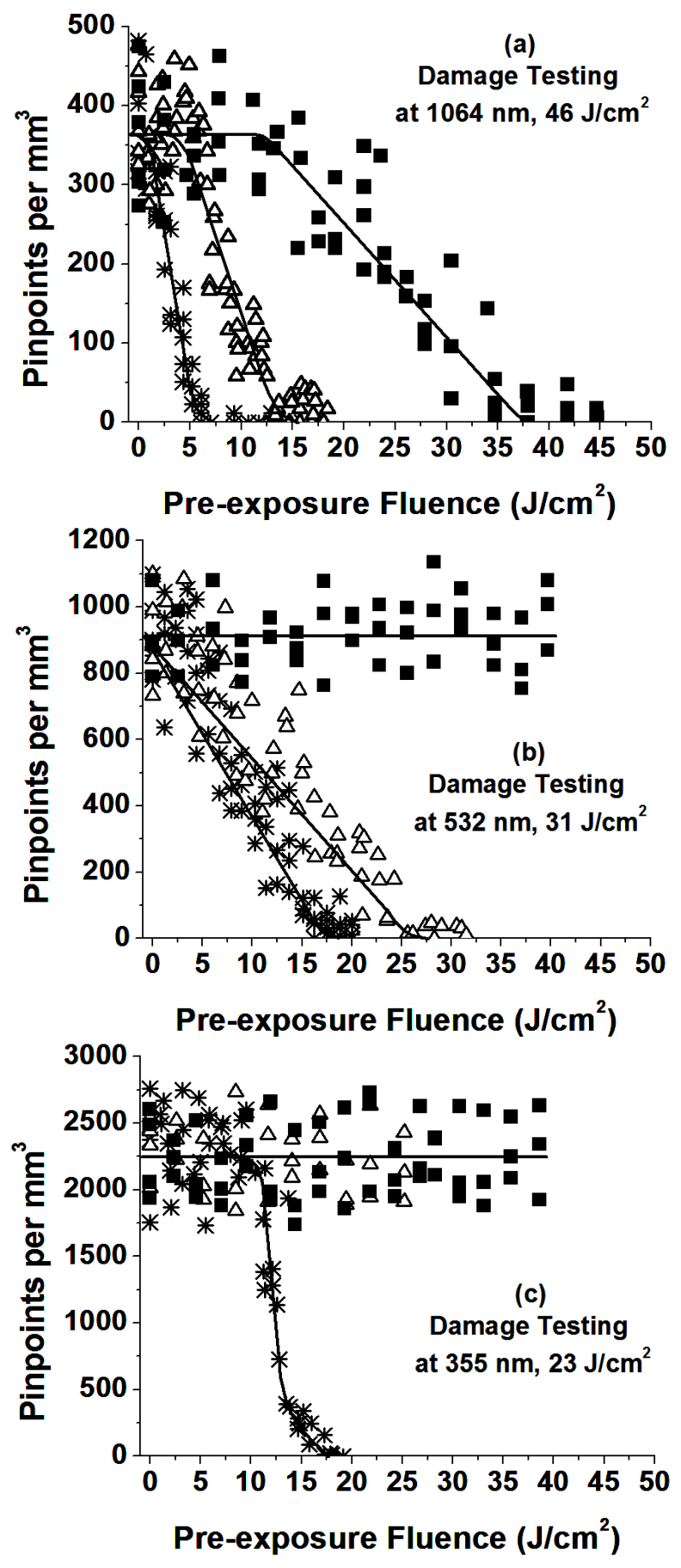

Figure 2. The density of new pinpoints resulting from damage testing pulse at a) $1064 \mathrm{~nm}$ at $46 \mathrm{~J} / \mathrm{cm}^{2}$, b) $532 \mathrm{~nm}$ at $31 \mathrm{~J} / \mathrm{cm}^{2}$, and c) $355 \mathrm{~nm}$ at $23 \mathrm{~J} / \mathrm{cm}^{2}$ as a function of the fluence of the ten pre-exposure pulses ( $\boldsymbol{\square}=$ pre-exposed to $1064 \mathrm{~nm},<=$ pre-exposed to 532 $\mathrm{nm}$, and $*$ = pre-exposed to $355 \mathrm{~nm}$ ) in DKDP. 
The density of new pinpoints resulting from damage testing at $\sim 46 \mathrm{~J} / \mathrm{cm}^{2}$ at $1064 \mathrm{~nm}$ for pre-exposure at each of the three harmonics is shown in Figure 2a. Without pre-exposure, the observed PPD from damage testing is $\sim 380 \mathrm{pp} / \mathrm{mm}^{3}$. Following pre-exposure at $355 \mathrm{~nm}$ the results show that the density of new pinpoints after damage testing rapidly decreases with increase in pre-exposure fluence starting from the lowest tested pre-exposure fluence at $\sim 0.7 \mathrm{~J} / \mathrm{cm}^{2}$. For preexposure fluence of $\sim 6 \mathrm{~J} / \mathrm{cm}^{2}$, the density of new pinpoints decreases to almost $\sim 0 \mathrm{pp} / \mathrm{mm}^{3}$ (from the original $\sim 380$ $\mathrm{pp} / \mathrm{mm}^{3}$ ) while the pre-exposure is still below the damage threshold of the material (located at $\sim 8 \mathrm{~J} / \mathrm{cm}^{2}$ ). Pre-exposure at $532 \mathrm{~nm}$ shows no conditioning until $\sim 6 \mathrm{~J} / \mathrm{cm}^{2}$, demonstrating the presence of a threshold fluence for conditioning. A linear decrease in the density of new pinpoints in then observed with further increase in pre-exposure fluence. Damage testing results in very little or no additional damage for pre-exposure of $\sim 14 \mathrm{~J} / \mathrm{cm}^{2}$ and above but this pre-exposure fluence is above the damage threshold of the material (located at $\sim 12 \mathrm{~J} / \mathrm{cm}^{2}$ ). Pre-exposure at $1064 \mathrm{~nm}$ also shows that a threshold pre-exposure fluence exists for conditioning located at $\sim 13 \mathrm{~J} / \mathrm{cm}^{2}$ followed by a linear decrease in the PPD. The density of new pinpoints decreases to $\sim 0 \mathrm{pp} / \mathrm{mm}^{3}$ with pre-exposure at $\sim 40 \mathrm{~J} / \mathrm{cm}^{2}$, well above the damage threshold at $1064 \mathrm{~nm}$ which is at $\sim 24 \mathrm{~J} / \mathrm{cm}^{2}$.

Figure $2 \mathrm{~b}$ shows the three sets of data for damage testing at $\sim 31 \mathrm{~J} / \mathrm{cm}^{2}$ at $532 \mathrm{~nm}$ in material pre-exposed at each of the three harmonics. Damage testing without pre-exposure provides $\sim 900 \mathrm{pp} / \mathrm{mm}^{3}$. With pre-exposure at $355 \mathrm{~nm}$,.the density of new pinpoints from damage testing appears to decrease linearly with increase in pre-exposure fluence starting from the lowest tested pre-exposure fluence. For pre-exposure above $\sim 18 \mathrm{~J} / \mathrm{cm}^{2}$, almost no new pinpoints are observed from damage testing. Similarly, pre-exposure at $532 \mathrm{~nm}$ results in a linear decrease in the density of new pinpoints, however, the best conditioning occurs at a much higher pre-exposure fluence of $\sim 32 \mathrm{~J} / \mathrm{cm}^{2}$. Damage testing following pre-exposure at $1064 \mathrm{~nm}$ up to $\sim 40 \mathrm{~J} / \mathrm{cm}^{2}$ indicates no improvement to the damage performance.

Figure $2 \mathrm{c}$ shows the three data sets corresponding to the damage testing at $\sim 23 \mathrm{~J} / \mathrm{cm}^{2}$ at $355 \mathrm{~nm}$ and pre-exposure at each harmonic. Damage testing without pre-exposure provides $\sim 2250 \mathrm{pp} / \mathrm{mm}^{3}$. Similar to the behavior observed for damage testing at $1064 \mathrm{~nm}$ and pre-exposure at both 1064 and $532 \mathrm{~nm}$ (shown in Fig. 2a), pre-exposure at $355 \mathrm{~nm}$ demonstrates the presence of a threshold fluence for conditioning at $\sim 10 \mathrm{~J} / \mathrm{cm}^{2}$. However, the density of new pinpoints then rapidly decreases with increasing fluence. Pre-exposure at 532 and $1064 \mathrm{~nm}$ up to fluences of $\sim 25 \mathrm{~J} / \mathrm{cm}^{2}$ and $\sim 38$ $\mathrm{J} / \mathrm{cm}^{2}$, respectively, indicate no improvement to the damage performance.

The results show three different characteristic behaviors. For damage testing at $1064 \mathrm{~nm}$, a "threshold" fluence for conditioning is observed followed by a linear decrease in damage density at higher pre-exposure fluence. For damage testing at $532 \mathrm{~nm}$, a linear behavior is observed with no noticeable threshold fluence. For damage testing at $355 \mathrm{~nm}$, a sharp nonlinear or "step-threshold" behavior is the dominant feature. Elsewhere in this proceedings experimental results have indicated that the defects responsible for damage initiation at 532 and $355 \mathrm{~nm}$ are the same and the defects responsible for damage initiation at $1064 \mathrm{~nm}$ are different (to those at 532 and $355 \mathrm{~nm}$ ). ${ }^{2,3}$ These different behaviors, if arising from the same type of defects responsible for damage initiation, suggest the presence of multiple pathways leading to conditioning.

\subsection{Conditioning vs. Damage Testing and Pre-exposure Fluence}

In order to learn the extent of laser conditioning in improving damage performance of these materials one needs to also consider the dependence of the conditioning threshold of individual defects on their damage initiation threshold. More specifically, is there a specific conditioning threshold for all 355-nm damage initiating defects or is this threshold different for each initiator? If the latter is true, then does the conditioning threshold of each initiator relate to the individual damage initiation threshold which may tie both processes to a characteristic property of the initiators (e.g. size or density)? These questions may be hard to address but some insight can be offered by studying the damage behavior at different laser fluences using the same conditioning protocol used above. Specifically, experiments were performed to investigate the conditioning effectiveness versus pre-exposure fluence for two damage testing fluences with both preexposure and damage testing at $355 \mathrm{~nm}$. In one case the damage testing fluence was $15-16 \mathrm{~J} / \mathrm{cm}^{2}$ while in the other case the damage testing fluence was $20-21 \mathrm{~J} / \mathrm{cm}^{2}$. In both cases sites were exposed to a single damage testing pulse following exposure to ten pulses of the same (pre-exposure) fluence.

Figure 3 shows the density of new pinpoints resulting from damage testing as a function of the pre-exposure fluence, for both experiments. The results of damage testing at $20-21 \mathrm{~J} / \mathrm{cm}^{2}$ at $355 \mathrm{~nm}$ are shown as solid circles and the results of damage testing at $15-16 \mathrm{~J} / \mathrm{cm}^{2}$ at $355 \mathrm{~nm}$ are shown as open circles. The results show that the conditioning threshold in 
both experiments starts at $\sim 6.5 \mathrm{~J} / \mathrm{cm}^{2}$. A drop of $\sim 1000$ pinpoints per $\mathrm{mm}^{3}$ for damage testing at both $15-16 \mathrm{~J} / \mathrm{cm}^{2}$ and $20-21 \mathrm{~J} / \mathrm{cm}^{2}$ occurs at pre-exposure fluences between $\sim 6.5 \mathrm{~J} / \mathrm{cm}^{2}$ and $\sim 10 \mathrm{~J} / \mathrm{cm}^{2}$ (depicted in Fig. 3). This demonstrates that the population of conditioned defects for this pre-exposure, equivalent to $\sim 1000$ pinpoints per $\mathrm{mm}^{3}$, is independent of the damage testing fluence. Moreover, this pre-exposure only conditions a subset of the defect population. The population of damage initiators that initiates damage at $15-16 \mathrm{~J} / \mathrm{cm}^{2}$ has been mostly diminished (some initiators erupt to form damage sites during pre-exposure). One then may argue that the defects that initiate damage at $15-16 \mathrm{~J} / \mathrm{cm}^{2} \mathrm{can}$ be conditioned with pre-exposure fluences at $10 \mathrm{~J} / \mathrm{cm}^{2}$. The PPD profile obtained with damage testing at $20-21 \mathrm{~J} / \mathrm{cm}^{2}$ shows that further pre-exposure up to $17 \mathrm{~J} / \mathrm{cm}^{2}$ reduces the PPD by another $\sim 1300$ pinpoints per $\mathrm{mm}^{3}$ indicating that an additional population of defects condition at a higher fluence. This result indicates that pre-exposure at a fixed fluence at $355 \mathrm{~nm}$ conditions only a portion of the whole population of defects leading to damage initiation at $355 \mathrm{~nm}$ and has no effect on the remaining defects.

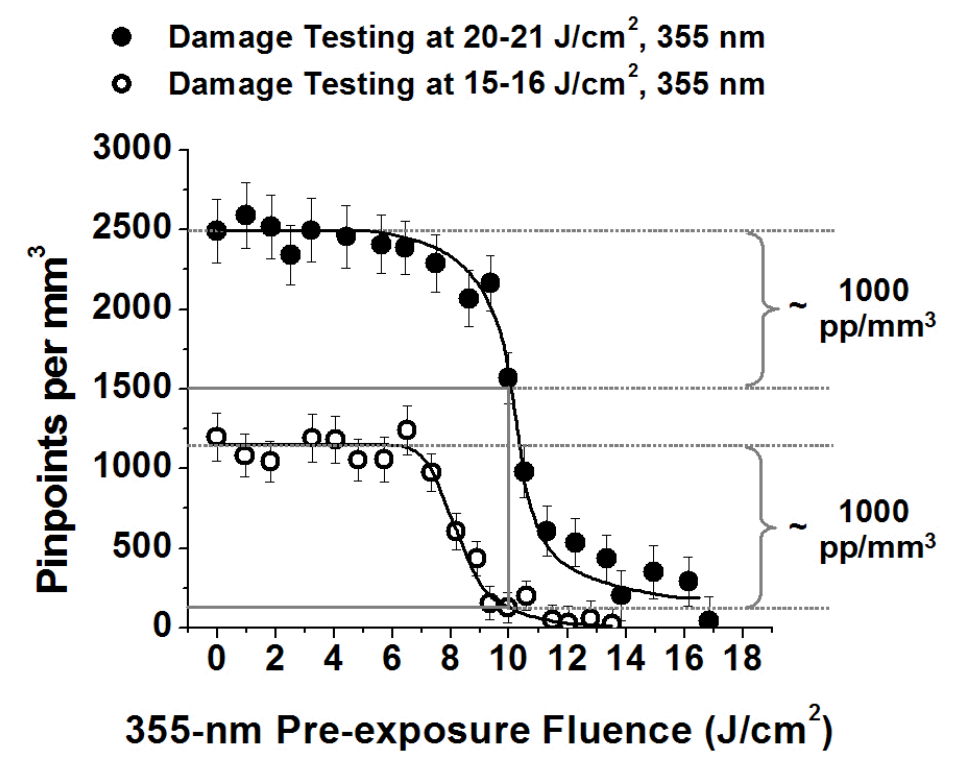

Figure 3. The total PPD resulting from both damage testing and pre-exposure to ten pulses at $355 \mathrm{~nm}$ as a function of the pre-exposure fluence. $\mathbf{O}=$ damage testing at $15-16 \mathrm{~J} / \mathrm{cm}^{2}$ and $\mathbf{O}=$ damage testing at $20-21 \mathrm{~J} / \mathrm{cm}^{2}$.

\subsection{Conditioning vs. Pre-exposure Number of Pulses and Total Energy Using Ramping Method}

The damage performance at $355 \mathrm{~nm}$ of material conditioned at $355 \mathrm{~nm}$ using the ramped-fluence pre-exposure method was explored, looking specifically at the dependence of conditioning on the fluence step size and number of preexposure pulses per step in the ramping protocol. This experiment provides information on the optimal ramping fluence protocol using the smallest level of exposure (i.e., the largest fluence step sizes and the least number of pulses per step) as well as the dependence of conditioning of the defects responsible for damage on the total pre-exposure energy and intensity. Pristine bulk sites were exposed to gradually increasing (i.e., ramped) peak laser fluences in $1-$ or $2-\mathrm{J} / \mathrm{cm}^{2}$ steps starting at $1 \mathrm{~J} / \mathrm{cm}^{2}$. At each fluence step, the material was exposed to a fixed number of pulses $(1,2,5$ or 10 pulses). The fluence was ramped up to the highest fluence at which no damage would be observed using the CCD camera. Sites within the ramp-pre-exposed volume were then exposed to single damage testing pulses at various fluences and the PPD was measured.

Figure 5 shows the PPD resulting from damage testing as a function of the testing fluence for several ramped preexposure protocols. The ramping pre-exposure protocol corresponding to each damage density profile is simplified using the following functional description: $\mathrm{P}\left(\right.$ ramping fluence step in $\mathrm{J} / \mathrm{cm}^{2}$, \# of pulses at each step, maximum fluence reached during ramping without damaging). The results show that the onset of damage in the pristine material is $\sim 5 \mathrm{~J} / \mathrm{cm}^{2}$. A 
significant increase to the fluence onset of damage (to $\sim 7 \mathrm{~J} / \mathrm{cm}^{2}$ from $\sim 5 \mathrm{~J} / \mathrm{cm}^{2}$ in the pristine material) and the overall damage performance is observed with ramping pre-exposure using as large of a fluence step as $2 \mathrm{~J} / \mathrm{cm}^{2}$ and as little as 1 pulse per step, demonstrated by the $\mathrm{P}(2,1,5)$ damage profile. The $\mathrm{P}(2,2,5)$ profile shows that the increase in number of pulses from 1 to 2 per fluence step provides no increase to neither the maximum fluence reached during pre-exposure nor the level of conditioning. The $\mathrm{P}(1,1,7)$ and $\mathrm{P}(2,10,7)$ damage profiles are similar, both showing a significant increase to the fluence onset of damage to $\sim 9 \mathrm{~J} / \mathrm{cm}^{2}$ and overall damage performance. Their comparison suggests that the smaller step size of the $\mathrm{P}(1,1,7)$ profile compensates for the greater number of pulses in the $\mathrm{P}(2,10,7)$, although both reach the same maximum fluence of $7 \mathrm{~J} / \mathrm{cm}^{2}$ during ramping pre-exposure. The $\mathrm{P}(1,2,8)$ profile shows that the increase in number of pulses per step to 2 from 1 in the $\mathrm{P}(1,1,7)$ profile results both in a higher maximum ramping pre-exposure fluence and better conditioning (a fluence onset of damage of $\sim 10 \mathrm{~J} / \mathrm{cm}^{2}$ compared to $\sim 9 \mathrm{~J} / \mathrm{cm}^{2}$ ). The $\mathrm{P}(1,10,9)$ profile reaches the highest maximum ramping pre-exposure fluence and shows an increase to the fluence onset of damage to $\sim 11 \mathrm{~J} / \mathrm{cm}^{2}$. An additional 1000 pulses at $9 \mathrm{~J} / \mathrm{cm}^{2}$ on top of the $\mathrm{P}(1,10,9)$ ramping protocol shows very little improvement to the overall damage performance and only $\sim 1 \mathrm{~J} / \mathrm{cm}^{2}$ increase to the fluence onset of damage from the $\mathrm{P}(1,10,9)$ only ramping protocol.

The results show that conditioning becomes increasingly better with smaller ramping fluence steps and greater numbers of pulses. However, we observe a direct correlation between the relative level of conditioning and the maximum fluence reached during ramping pre-exposure without damaging the material. Moreover, the maximum fluence becomes higher with smaller ramping fluence steps and greater numbers of pulses. With the addition of 1000 pulses to the $\mathrm{P}(1,10,9)$ protocol at the maximum ramping pre-exposure fluence of $9 \mathrm{~J} / \mathrm{cm}^{2}$ there is very little additional improvement to the overall damage performance compared to the $\mathrm{P}(1,10,9)$ protocol only. This demonstrates that the fluence step size or number of pulses per step individually is not the important factor but that the maximum ramping preexposure reached is the critical parameter in providing the best level of conditioning. In this material, an approximately $2 \%$ increase to the damage performance can be achieved with ramping pre-exposure with fluence steps as large as 1 $\mathrm{J} / \mathrm{cm}^{2}$ and as few pulses as 10 per fluence step.

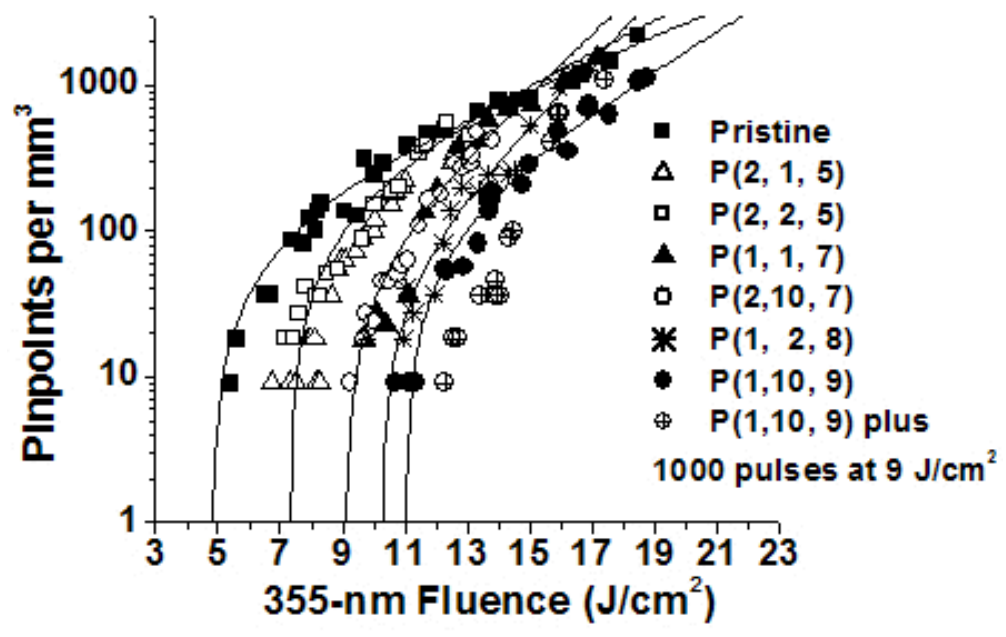

Figure 5: Damage density profiles at $355 \mathrm{~nm}$ in DKDP on a log-linear scale without and with conditioning using various fluence ramping protocols. The function that corresponds to each damage density profile describes the ramping protocol as follows: $\mathrm{P}(\mathrm{ramping}$ fluence step in $\mathrm{J} / \mathrm{cm}^{2}$, \# of pulses at each step, maximum fluence reached during ramping without damaging). The lines shown are the best fits to each data set.

\subsection{Conditioning vs. Testing and Pre-exposure Wavelength Using Ramping Method}

Ramped-fluence pre-exposure was used as a method to explore optimal improvement to the damage performance at all of the nine combinations of damage testing and pre-exposure at 1064, 532, and $355 \mathrm{~nm}$. In addition to learning the level of conditioning that may be achieved and the necessary pre-exposure wavelengths for providing this optimal conditioning for operation at a specific wavelength, we also gain insight into the wavelength absorption characteristics 
leading to conditioning of the defects responsible for damage. The best performing pre-exposure fluence ramping protocol from the above results was used (i.e., $\mathrm{P}(1,10, \mathrm{X})$ protocol or exposing pristine bulk volumes to gradually increasing fluence levels in $1 \mathrm{~J} / \mathrm{cm}^{2}$ steps starting at $1 \mathrm{~J} / \mathrm{cm}^{2}$ ). The volumes were rastered by setting the sample stage speed such that each volume was exposed to 10 pulses at peak fluence at each fluence step. The fluence was ramped up to the highest fluence at which no damage was observed by the CCD camera. This highest conditioning fluence was approximately $11 \mathrm{~J} / \mathrm{cm}^{2}$ at $355 \mathrm{~nm}, 17 \mathrm{~J} / \mathrm{cm}^{2}$ at $532 \mathrm{~nm}$, and $24 \mathrm{~J} / \mathrm{cm}^{2}$ at $1064 \mathrm{~nm}$. The conditioned volume was then tested with single damage testing pulses with the fluence varying at each new site. An image of the damage was then captured for measurement of the resulting PPD.

Figure 4 shows data profiles of the PPD as a function of fluence, for damage testing at 1064, 532 and $355 \mathrm{~nm}$. These profiles demonstrate the damage behavior of a DKDP sample both without and with conditioning at each harmonic using the fluence-ramping method. The plots are on a semi-log scale and data fit lines are drawn in as a guide to the eye. The PPD profiles obtained under 1064-nm damage testing are shown in Fig. 1a. The observed onset of damage of the unconditioned material is found to be $\sim 20 \mathrm{~J} / \mathrm{cm}^{2}$. Pre-exposure at $1064 \mathrm{~nm}$ demonstrates a substantial increase in the onset of damage to $30 \mathrm{~J} / \mathrm{cm}^{2}$. Pre-exposure at $532 \mathrm{~nm}$ increases the onset of damage even more to $\sim 37.5 \mathrm{~J} / \mathrm{cm}^{2}$, and 355 $\mathrm{nm}$ pre-exposure shows at least $2 \times$ increase. The PPD profiles obtained under 532-nm damage testing are shown in Fig. $1 \mathrm{~b}$. The onset of damage of the unconditioned material is found to be $\sim 10 \mathrm{~J} / \mathrm{cm}^{2}$. An increase in the onset of damage to $15 \mathrm{~J} / \mathrm{cm}^{2}$ is achieved by pre-exposure at $355 \mathrm{~nm}$ while pre-exposure at $532 \mathrm{~nm}$ shows a larger increase in the onset of damage to nearly $19 \mathrm{~J} / \mathrm{cm}^{2}$. The PPD profiles obtained under $355-\mathrm{nm}$ damage testing are shown in Fig. 1c. These results show that pre-exposure at $355 \mathrm{~nm}$ increased the onset of damage to above $12.5 \mathrm{~J} / \mathrm{cm}^{2}$ from the $\sim 8 \mathrm{~J} / \mathrm{cm}^{2}$ measured in the unconditioned material. However, pre-exposure at $1064 \mathrm{~nm}$ and $532 \mathrm{~nm}$ results in no noticeable increase in the $355-\mathrm{nm}$ onset of damage.

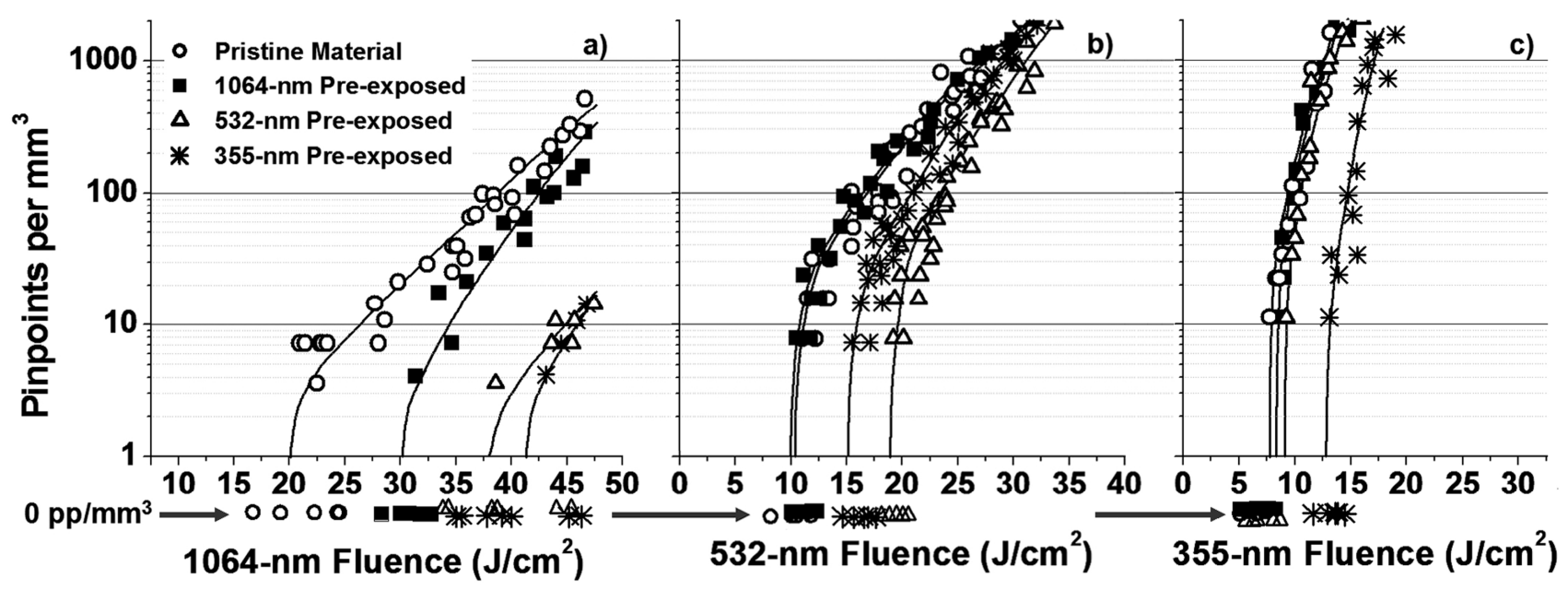

Figure 4. PPD profiles for a DKDP crystal sample on a semi-log scale at a) 1064, b) 532, and c) $355 \mathrm{~nm}$. The testing fluences for which no damage was observed (i.e., $0 \mathrm{pp} / \mathrm{mm}^{3}$ ) are indicated by the arrow. The curves are a guide to the eye.

As a general rule, conditioning occurs for pre-exposure at wavelengths equal to or shorter than that used for damage testing. Figure $4 \mathrm{~b}$ shows that pre-exposure to $532-\mathrm{nm}$ pulses provides better conditioning for $532-\mathrm{nm}$ operation than preexposure to 355-nm pulses, an opposite trend to that observed in Fig. 4a, where the lower wavelengths provided better conditioning for 1064-nm testing. This is likely due to the ability to reach higher fluence during pre-exposure at $532 \mathrm{~nm}$. Damage testing at $355 \mathrm{~nm}$ indicated that pre-exposure at either $1064 \mathrm{~nm}$ or $532 \mathrm{~nm}$ provides no improvement to damage resistance while, in contrast, pre-exposure at $355 \mathrm{~nm}$ shows significant improvement.

\subsection{Conditioning shelf lifetime}


Understanding the effectiveness of conditioning over time is a concern with regard to its application to KDP/DKDP optics in large-aperture laser systems. We performed an experiment to assess the damage performance of bulk DKDP without and with conditioning over a period of $\sim 6$ months. The conditioning was performed with both 3 -ns and $800-p s$ flat-top 355-nm pulses on LLNL's OSL facility. These pulse-lengths were used in order to gather data at two points within the pulse-length region determined to provide significant conditioning. ${ }^{4}$ The laser beam was $\sim 1 \mathrm{~cm}$ in diameter and two separate regions of the sample were exposed to ramping fluence at each pulse-length. The damage testing was performed with 3-ns pulses using the system described in this work. Damage density profiles of the pristine material and both conditioned regions were measured 4 days, 34 days, and 191 days after conditioning.

Figure 6 shows the PPD resulting from single 3-ns damage testing pulses at various fluences in the pristine, 3-ns ramp-conditioned, and 800-ps ramp-conditioned DKDP. The damage profiles of the pristine material are indicated by squares, the 3 -ns conditioned material by circles, and the 800 -ps material by triangles. The filled shapes indicate testing 4 days after conditioning, the unfilled shapes after 34 days, and the shapes with an "X" after 191 days. The onset of damage of the pristine material takes place at $\sim 5 \mathrm{~J} / \mathrm{cm}^{2}$. Four days after conditioning, the onset of damage occurs at fluences of $\sim 7 \mathrm{~J} / \mathrm{cm}^{2}$ and $\sim 9 \mathrm{~J} / \mathrm{cm}^{2}$ for the 3-ns and 800-ps conditioned regions, respectively. The damage performance of the 3-ns and 800-ps conditioned materials begin to converge with that of the pristine material at higher PPDs. Specifically, the PPD profile of the 3-ns conditioned material overlaps with that of the pristine material for PPDs of $\sim 1000 \mathrm{pp} / \mathrm{mm}^{3}$ and higher. In this PPD range, the PPD profile of the 800-ps conditioned material is still below that of the pristine material with the profiles appearing to convergence at higher PPDs. The damage profiles of the material after 34 and 191 days indicate no change to the damage performance of either conditioned regions.

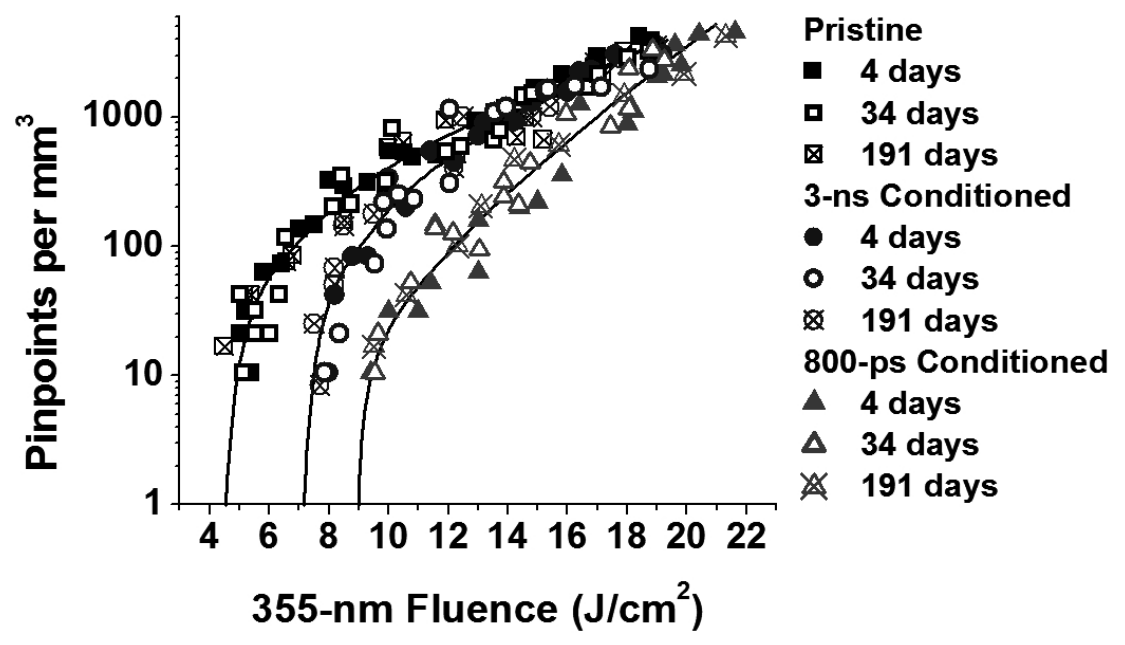

Figure 6. Damage density profiles on log-linear scale in a DKDP sample without conditioning (shown as squares), with conditioning at $3 \mathrm{~ns}$ (shown as circles), and 800 ps (shown as triangles) measured after 4 days (solid shapes), 34 days (unfilled shapes), and 191 days (shapes with an "X"). The three curves are fits to the three profiles measured after 4 days, to guide the eye.

\section{CONCLUSION}

The ramp conditioning results show that the level of conditioning achieved at $355 \mathrm{~nm}$ depends on the maximum fluence that can be reached during conditioning without causing damage. This maximum fluence depends on the ramping fluence step size and number of conditioning pulses. Furthermore, conditioning is demonstrated to take place using wavelengths at or below the testing wavelength. The results of investigating conditioning as a function of the preexposure fluence reveal that a "threshold" pre-exposure fluence is necessary when conditioning for 355-nm operation while, on the other hand, a linear dependence of conditioning on pre-exposure fluence is observed when conditioning for 532-nm operation. These different behaviors arising from conditioning of the same set of initiators suggest that there may be more than one conditioning pathway in KDP crystals that depend on the laser intensity and wavelength of the 
conditioning pulses. Damage testing and conditioning at different fluences at $355 \mathrm{~nm}$ suggests that pre-exposure at a fixed fluence conditions only a subset of the defect population, instead of improving the damage behavior of the entire population of damage initiating defects.

\section{ACKNOWLEDGEMENTS}

This work was performed under the auspices of the U.S. Department of Energy by University of California, Lawrence Livermore National Laboratory under contract W-7405-Eng-48.

\section{REFERENCES}

1. P. DeMange, C. W. Carr, H. B. Radousky, and S. G. Demos, "System for evaluation of laser-induced damage performance of optical materials for large aperture lasers," Rev. Sci. Instrum. 75, 3298 (2004).

2. R. A. Negres, P. DeMange, H. B. Radousky, S. G. Demos, "Laser-Induced Damage in DKDP Crystals under Simultaneous Exposure to Laser Harmonics," in these proceedings 5991-30.

3. P. DeMange, R. A. Negres, H. B. Radousky, S. G. Demos, "Different precursor populations revealed by microscopic studies of bulk damage in KDP and DKDP crystals," in these proceedings 5991-87.

4. J. J. Adams, T. L. Weiland, J. R. Stanley, W. D. Sell, R. L. Luthi, J. L. Vickers, C. W. Carr, M. D. Feit, A. M. Rubenchik, M. L. Spaeth, and R. P. Hackel, "Pulse-length dependence of laser conditioning and bulk damage in $\mathrm{KD}_{2} \mathrm{PO}_{4}$," in Laser-Induced Damage in Optical Materials: 2004, Gregory J. Exarhos, Arthur H. Guenther, Norbert Kaiser, Keith L. Lewis, M. J. Soileau, Christopher J. Stolz, Eds., Proc. SPIE 5647, 265-278, 2005. 